\title{
A Program of Physical Rehabilitation during Hemodialysis Sessions Improves the Fitness of Dialysis Patients
}

\author{
Tomasz Gołębiowski ${ }^{a}$ Mariusz Kusztal ${ }^{a}$ Wacław Weyde ${ }^{a}$ Wioletta Dziubek ${ }^{b}$ \\ Marek Woźniewski ${ }^{b}$ Katarzyna Madziarska ${ }^{a}$ Magdalena Krajewska ${ }^{a}$ \\ Krzysztof Letachowicz $^{a} \quad$ Beata Strempska $^{a}$ Marian Klinger ${ }^{a}$ \\ ${ }^{a}$ Department of Nephrology and Transplantation Medicine, Medical University, and ${ }^{b}$ Faculty of Physiotherapy, \\ University School of Physical Education, Wroclaw, Poland
}

\section{Key Words}

End-stage renal disease $\cdot$ Rehabilitation • Capacity •

Physical training $\cdot$ Dialysis

\begin{abstract}
Aim: The aim of the present study was to evaluate the influence of cycle exercise during hemodialysis (HD) on patients' physical proficiency, muscle strength, quality of life and selected laboratory parameters. Patients and Methods: In a group of 29 (15 female, 14 male) HD patients (age $64.2 \pm 13.1$ years), 3 months of cycle training during dialysis sessions was performed. The following data were analyzed: strength of lower extremities (six-minute walk test, isokinetic knee extension, flexion peak torque), nutrition parameters (albumin, BMI), inflammation intensity (CRP, IL-6), and quality of life (SF36v2). Results: In the six-minute walk test, the increase in walk velocity was $4 \%(3.56 \mathrm{~km} / \mathrm{h}$ before and $3.73 \mathrm{~km} / \mathrm{h}$ after cycle training; $p<0.01$ ). At angular velocity (AV) of $60 \%$, extension peak torque in the knee joint rose by $7 \%$ and at AV of $300 \%$ by $4 \%(p=0.04)$. Flexion peak torque at AV of $180 \%$ increased by $13 \%(p=0.0005)$. The program does not influ-
\end{abstract}

ence nutrition or inflammation parameters. No complications directly related to exercise were observed. Conclusion: Cycle exercise during dialysis is safe even in older HD patients with multiple comorbidities. It results in a significant increase in general patient walking ability and in a gain in lower extremity muscle strength.

Copyright @ 2012 S. Karger AG, Basel

\section{Introduction}

Patients with end-stage renal disease (ESRD) receiving maintenance dialysis have more limited physical function than age-matched healthy people. This has been shown in several studies which assessed walking speed [1], strength [2] and quality of life [3]. Many factors may contribute to this disturbance: anemia [4], the uremic milieu itself, inflammatory state and malnutrition [5].

O'Hare et al. [6] reported that more than one third of this population never or almost never engage in exercise activity. That is why a sedentary lifestyle is considered a leading cause of low physical capacity. Deterioration of

\section{KARGER}

Fax +4161306 1234

E-Mail karger@karger.ch

www.karger.com (c) 2012 S. Karger AG, Basel

$1420-4096 / 12 / 0354-0290 \$ 38.00 / 0$

Accessible online at:

www.karger.com/kbr
Tomasz Gołębiowski

Department of Nephrology and Transplantation Medicine

Medical University, ul. Borowska 213

PL-50-556 Wroclaw (Poland)

Tel. +48 7173325 00, E-Mail tgolebiowski@op.pl 
Fig. 1. Exercise during HD.

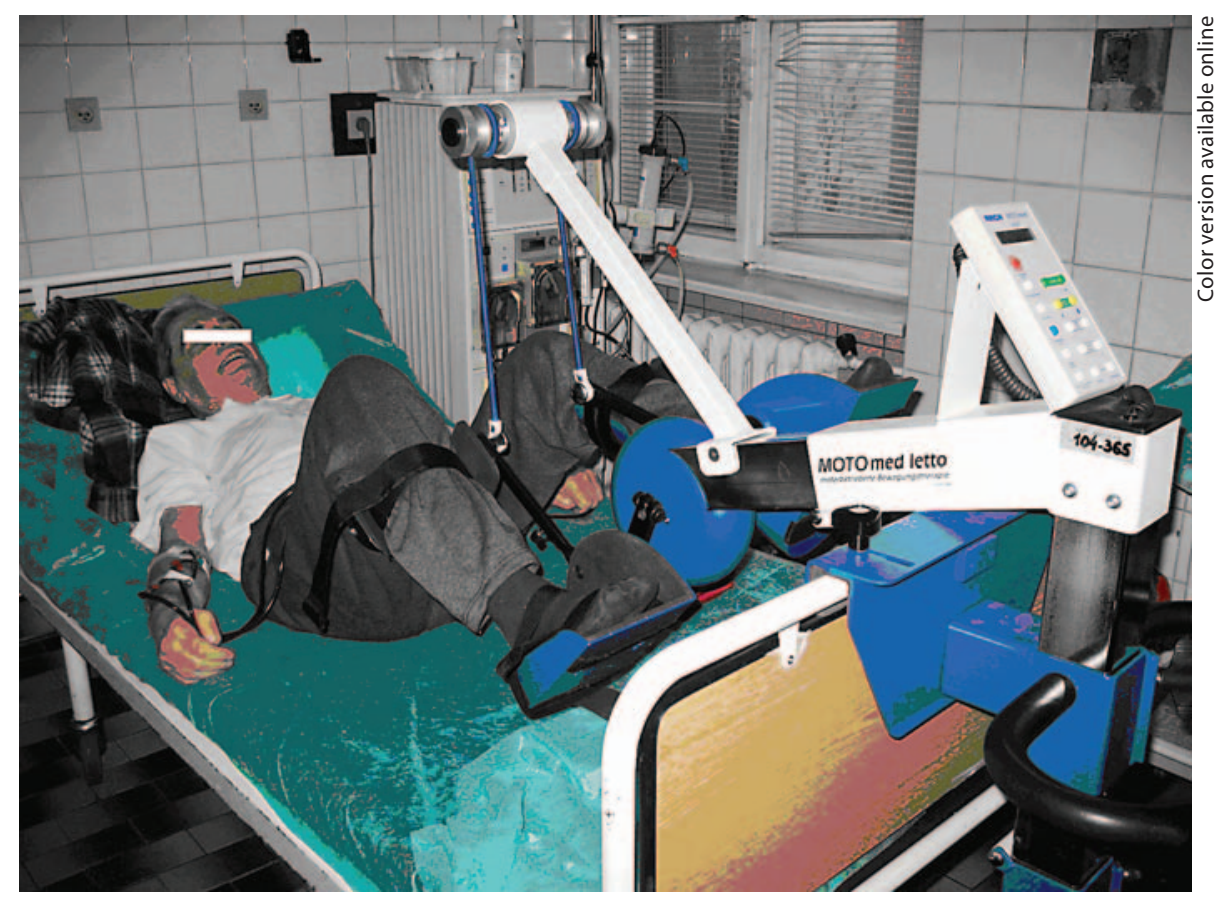

physical function observed in dialysis patients is associated with development of disability, low work capacity and poor perception of quality of life [7]. Additionally, in older patients, loss of self-reliance and even ability to selfcare present one of the important socioeconomic problems. This is why specific exercise programs focused on physical function are recommended to make them more independent.

The positive influence of physical activity on physical fitness is well known in the general population. Taking it into account, it should be feasible to achieve a comparable benefit in the group of ESRD patients. Because of many reasons (fear of adverse events, limited time, a belief that patients would not follow a suggestion to increase activity) nephrologists do not assess patients' physical activity and do not give counsel to increase their activity, but even the smallest effort in this regard could bring significant improvement [8].

We implemented 3 months of cycle exercise during hemodialysis (HD) to study its influence on patients' physical proficiency and muscle strength of the lower extremities. Additionally, we evaluated safety of the exercise during HD sessions and its impact on quality of life, nutritional parameters, inflammation intensity and antihypertensive treatment.

Rehabilitation during Hemodialysis
Table 1. Sensitivity, precision and normal values of inflammatory cytokine assays in healthy volunteers

\begin{tabular}{lcll}
\hline & $\begin{array}{l}\text { Sensitivity } \\
(\mathrm{MDD}), \mathrm{pg} / \mathrm{ml}\end{array}$ & $\begin{array}{l}\text { Precision } \\
(\mathrm{CV}), \%\end{array}$ & $\begin{array}{l}\text { Normal } \\
\text { values, } \mathrm{pg} / \mathrm{ml}\end{array}$ \\
\hline IL-1 $\beta$ & $<1$ & $2.8-8.5$ & $<3.9$ \\
IL-1ra & $2.15-18.3$ & $3.7-11$ & $360 \pm 214$ \\
IL-6 & $<0.7$ & $1.6-6.4$ & $<12.5$ \\
TNF & $0.5-5.5$ & $4.2-7.4$ & $<15.6$ \\
\hline
\end{tabular}

$\mathrm{MDD}=$ Minimum detectable dose $\mathrm{CV}=$ coefficient of variation.

\section{Patients and Methods}

Patients

Twenty-nine patients (15 men and 14 women; mean age 64.2 \pm 13.1 years; $4-192$ months on HD maintenance) were recruited from the outpatient HD unit of the Department of Nephrology and Transplantation Medicine in Wroclaw. The inclusion criteria were as follows: (1) patients who had undergone dialysis treatment for at least 3 months; (2) age older than 18 years, and (3) a hemoglobin level $>8 \mathrm{~g} / \mathrm{dl}$. Patients with the following conditions were excluded: (1) uncontrolled heart failure; (2) evident infection; (3) poorly controlled hypertension, and (4) uncontrolled arrhythmia. The study protocol was approved by the Eth-

Kidney Blood Press Res 2012;35:290-296 
ics Committee of the Medical University of Wroclaw. Written informed consent was obtained from all patients. The baseline characteristics of the study patients are displayed in table 1 . The 3 -month program of exercises was completed by 21 of 29 patients (72.4\%). Six patients withdrew their consent in the third week of the program, announcing that exercises would not affect their well-being and therefore they did not want to continue it. One person with a severe form of uremic neuropathy resigned from the program because of exacerbation of the symptoms while performing exercises, and another one discontinued due to problems with an arteriovenous fistula (AVF) needing surgical correction.

\section{Methods}

\section{Exercise during HD}

Patients exercised three times weekly during dialysis sessions on MOTOmed letto equipment made by RECK MOTOmed ${ }^{\circledR}$. This device is easy to fix to a patient's bed and allows one to cycle in a lying position (fig. 1). It is possible to perform active and also passive exercise with the help of its own electrical motor. Total duration of the training was 3 months. Each time patients started exercising after the beginning of HD sessions and continued for $50 \pm 19 \mathrm{~min}$. The physical load was individually adapted to exercise tolerance. The energy expenditure during one session with exercise was diverse and amounted to $403 \pm 219 \mathrm{~kJ}$. This significant diversity of energy expenditure and exercise time reflects the heterogeneity of the study group in terms of muscle strength capability used in cycling.

Blood pressure and heart rate during exercise were monitored every 15 min by the automatic blood pressure manometer A\&D UA-767 made by A\&D Instruments Ltd. Exercise was terminated if (1) the patient requested it; (2) the pulse rate exceeded the value of $80 \%$ of the maximal heart rate [the maximal heart rate (beat/ $\min )=220$ - age (years)], and (3) fatigue of the legs occurred.

\section{Muscle Strength of Lower Extremities}

Before (M0) and after the 3 months (M3) of exercise, the maximal peak torque in the knee joint was assessed using the isokinetic digital dynamometer Multi Joint 3 - Biodex System in the laboratory of the Physiotherapy Faculty in Wroclaw. This is a noninvasive method that uses the isokinetic mode and enables reliable measurement. Both lower limbs were tested. Each time the patient was informed how to perform the task. The test relies on flexion and extension of the legs at the knee joint with maximal force and measuring maximal flexion and extension peak torque for various arc speeds: 60,180 , and $300 \%$ s (10 movements for each speed and 30-second rest between each set). Muscle strength of lower limbs was recorded in the computer memory.

\section{Six-Minute Walk Test}

The six-minute walk test (6MWT) was used as an index of physical capacity, and was also carried out twice - before and after the 3-month program of exercises. It has been shown to be reliable to establish the physical capacity of patients with heart [9] and respiratory insufficiency [10] and also in patients with chronic renal failure [11]. This test can be used to assess the effects of rehabilitation, particularly for those in whom maximal exercise testing is not possible, for example in older patients. It was performed on the day of dialysis before the HD session. $\mathrm{Pa}$ tients walked unassisted for a 25-meter distance of the hospital corridor, accompanied by a physiotherapist. Participants were permitted to stop and to have a rest. The distance of each patient was recorded in meters and as an average walking speed (kilometers per hour).

\section{Safety Issues}

To evaluate the safety of the exercise, the following complications and symptoms were registered: incidents of hyper- and hypotension, stenocardia, cardiac arrhythmias, dislocation of the dialysis needle and muscle cramps. Before and after dialysis with exercise, a blood sample was taken to assess potassium level, and blood gas analysis was performed to monitor possible electrolyte and acid-base disturbances.

\section{Quality of Life}

To evaluate self-reported quality of life, the Polish version of the Short Form 36-item (SF-36v2) questionnaire was used. This questionnaire has 36 items divided into eight scales: Physical functioning (PF), Role functioning/physical, Bodily pain, General health, Vitality, Social functioning, Role functioning/emotional, and Mental health. These scales are scored from 0 to 100. If a score is higher, the self-reported perception of health is better. The questionnaires were completed independently.

\section{Other Laboratory Tests}

Blood was drawn for biochemical parameters before or after midweek dialysis. hs-CRP, albumin and lipids were measured by standard methods. Blood samples for measurements of lipids were obtained after an overnight fast. Measurement of inflammatory cytokine activity (IL-6, IL-1 $\beta$, IL-1ra, TNF) was performed in serum using immunoassay of R\&D System and spectroscopic techniques. Assay sensitivity and precision and normal values of healthy volunteers are presented in table 1 . All blood samples for gas analysis parameters were taken directly from an AVF.

\section{Statistical Analysis}

Comparisons between groups of all clinical and biochemical parameters were made using Student's t test for dependent samples. In case of deviation from normal distribution, additionally nonparametric tests were performed (Fisher's sign test for dependent samples, Wilcoxon matched-pairs signed-rank test) to confirm significance of the results. Group data are represented as mean \pm SD (95\% confidence intervals). The differences were acknowledged as significant if $\mathrm{p}<0.05$. All analyses were performed using Statistica 8.0 software package.

\section{Results}

\section{Baseline Characteristics, Cause of ESRD and Comorbidities}

In general, the study group of patients mainly consists of elderly patients with mean age of 64 years and many comorbidities. In over $80 \%$ of patients, hypertension was present. In 52\%, coronary heart disease was diagnosed. Four patients experienced myocardial infarction in the 
past. The main cause of ESRD was hypertensive (ischemic) nephropathy (34\%; table 2 ).

\section{MWT and Strength of Lower Extremities}

During the 3-month exercise program, an improvement in strength of lower extremities was achieved. In $6 \mathrm{MWT}$, the increase in speed was $4 \%(3.56 \mathrm{~km} / \mathrm{h}$ before, $3.73 \mathrm{~km} / \mathrm{h}$ after the program, $\mathrm{p}=0.01)$. The maximal extension peak torque in the knee joint rose by 7\% (69.77 $\mathrm{Nm}$ before and $75.43 \mathrm{Nm}$ after cycle training, $\mathrm{p}=0.01)$ at an angular velocity of $60 \%$ and by $4 \%(42.50 \mathrm{Nm}$ before and $44.11 \mathrm{Nm}$ after cycle training, $\mathrm{p}=0.04)$ at an angular velocity of $300 \%$. There was also an increase in maximal flexion peak torque at an angular velocity of $180 \%$ of $13 \%$ (24.49 $\mathrm{Nm}$ before and 28.17 Nm after cycle training, $\mathrm{p}=$ 0.0005 ; table 3 ).

\section{Quality of Life (SF-36)}

There was no statistically significant change in quality of life categories, but in the subgroup of 7 patients with initially the lowest $\mathrm{PF}$ score ( $\mathrm{PF}<30$ points) a significant increase in PF score at the end of the study was observed (12.86 vs. 24.29 points, $\mathrm{p}=0.04$ ).

\section{Other Clinical and Laboratory Parameters}

Nutrition parameters (weight, BMI, albumin, lipids), $\mathrm{C}$-reactive protein and other inflammatory cytokines (IL-6, IL-1 $\beta$, IL-1ra, TNF) did not change through the 3 months of regular stationary cycling during HD. There were also no significant differences in systolic or diastolic blood pressure. The mean number of antihypertensive medications was lower at the end of the study (2.00 at the start vs. 1.57 at the end), but the differences were not significant $(\mathrm{p}=0.11$; table 4$)$.

\section{Side Effects}

During 540 dialyses performed with exercise, complications were observed in 12 (2\%) HD sessions. They were the following: one incident of hypotension $(<90 / 50 \mathrm{~mm}$ $\mathrm{Hg}$ ) in a patient with diabetes, 5 incidents of muscle cramps, 4 incidents of systolic hypertension (max. 205 $\mathrm{mm} \mathrm{Hg}$ ), and 3 incidents of diastolic hypertension $>100$ $\mathrm{mm} \mathrm{Hg}$ (max. $110 \mathrm{~mm} \mathrm{Hg}$ ). In those cases of complications, exercise was stopped and any necessary fluids (in hypotension) or antihypertensive drugs (in hypertension) were given.

Additionally, we analyzed serum potassium and arterial gas parameters. Exercise did not raise potassium level after dialysis. In one subject with AVF failure, metabolic acidosis and poor dialysis adequacy parameters
Table 2. Baseline characteristics of the study population

\begin{tabular}{lc}
\hline Baseline characteristics & \\
Age, years & $64.2 \pm 13.1$ \\
Females, \% & 48.3 \\
Body weight, kg & $70.9 \pm 15.28$ \\
BMI & $26.21 \pm 4.91$ \\
Months on dialysis & $4-192$ \\
Hemoglobin, g/dl & $11.07 \pm 1.57$ \\
Albumin, g/dl & $3.99 \pm 0.53$ \\
\hline Comorbidities & \\
Hypertension & $24(82.8)$ \\
Coronary heart disease & $15(52.7)$ \\
Myocardial infarction & $4(13.8)$ \\
Atherosclerosis of lower extremities & $8(27.6)$ \\
Stroke & $2(6.9)$ \\
Diabetes type I & $2(6.9)$ \\
Diabetes type II & $2(6.9)$ \\
Atrial fibrillation & $2(6.9)$ \\
Kidney transplantation & $2(6.9)$ \\
Cardio-stimulator & $2(6.9)$ \\
Chronic obstructive pulmonary disease & $1(3.5)$ \\
\hline Cause of renal insufficiency & \\
Hypertensive (ischemic) nephropathy & $10(34.5)$ \\
Glomerulonephritis & $7(24.1)$ \\
Nephrolithiasis & $4(13.8)$ \\
Polycystic kidney disease & $3(10.3)$ \\
Diabetic nephropathy & $2(6.9)$ \\
Analgesic nephropathy & $1(3.5)$ \\
Unknown & $1(3.5)$ \\
Postpartum renal injury & $1(3.4)$ \\
\hline & \\
& \\
\hline
\end{tabular}

Figures in parentheses indicate percentages.

were recognized during the program. This patient was referred for fistula correction and he was excluded from the study.

\section{Discussion}

As a novel finding, our study demonstrates that an exercise program can be effectively and successfully implemented in high-risk HD patients encompassing elderly subjects with a high burden of comorbidities (table 2). During the 3-month exercise program, an improvement in functioning of lower extremities was observed. Speed gain during 6MWT increased significantly, from 3.56 $\mathrm{km} / \mathrm{h}$ at baseline to $3.73 \mathrm{~km} / \mathrm{h}$ at the end of the project $(\mathrm{p}<0.05)$. This improvement is similar to that reported 
Table 3. Results of maximal peak torque in the knee joint and 6MWT before and after 3-month cycle training

\begin{tabular}{llll}
\hline & M0 & M3 & p \\
\hline Peak torque flexion $60^{\circ}, \mathrm{Nm}$ & $49.71 \pm 76.24$ & $40.76 \pm 18.90$ & 0.492 \\
Peak torque extension $60^{\circ}, \mathrm{Nm}$ & $69.77 \pm 34.24$ & $75.43 \pm 38.66$ & 0.010 \\
Peak torque flexion $180^{\circ}, \mathrm{Nm}$ & $24.49 \pm 13.19$ & $28.17 \pm 12.49$ & 0.0005 \\
Peak torque extension $180^{\circ}, \mathrm{Nm}$ & $48.39 \pm 21.58$ & $49.41 \pm 21.1$ & 0.434 \\
Peak torque flexion $300^{\circ}, \mathrm{Nm}$ & $24.68 \pm 9.45$ & $28.82 \pm 6.98$ & 0.004 \\
Peak torque extension $300^{\circ}, \mathrm{Nm}$ & $42.50 \pm 12.05$ & $44.11 \pm 11.38$ & 0.34 \\
6MWT, km/h & $3.56 \pm 4.7$ & $3.73 \pm 5.13$ & 0.01 \\
\hline
\end{tabular}

Table 4. Values of blood pressure and parameters of nutrition, lipid metabolism and inflammation

\begin{tabular}{lccc}
\hline & M0 & M3 & p \\
\hline BMI & $26.21 \pm 4.91$ & $26.15 \pm 5.18$ & 0.77 \\
Albumin, g/dl & $3.99 \pm 0.53$ & $3.97 \pm 0.25$ & 0.88 \\
Ch, mg/dl & $208.81 \pm 50.57$ & $208.95 \pm 53.82$ & 0.99 \\
HDL, mg/dl & $44.11 \pm 7.65$ & $43.67 \pm 9.40$ & 0.77 \\
LDL, mg/dl & $127.18 \pm 48.88$ & $121.18 \pm 49.80$ & 0.44 \\
TG, mg/dl & $226.67 \pm 154.62$ & $208.39 \pm 78.41$ & 0.55 \\
SBP, mm Hg & $136.11 \pm 17.61$ & $135.38 \pm 20.29$ & 0.88 \\
DBP, mm Hg & $74.75 \pm 10.75$ & $73.13 \pm 10.58$ & 0.48 \\
Antihypertensive drugs & $2 \pm 1.45$ & $1.57 \pm 1.43$ & 0.11 \\
hs-CRP, mg/l & $13.93 \pm 10.36$ & $16.03 \pm 16.30$ & 0.54 \\
IL-1 $\beta, p g / m l$ & $1.93 \pm 2.43$ & $3.53 \pm 7.71$ & 0.17 \\
IL-1ra, pg/ml & $672.15 \pm 440.36$ & $767.57 \pm 434.67$ & 0.07 \\
IL-6, pg/ml & $13.07 \pm 11.13$ & $14.48 \pm 10.94$ & 0.28 \\
TNF, pg/ml & $5.66 \pm 3.14$ & $4.53 \pm 2.34$ & 0.12 \\
\hline
\end{tabular}

$\mathrm{Ch}=$ Cholesterol; $\mathrm{HDL}=$ high density lipoprotein LDL = low density lipoprotein; $\mathrm{TG}=$ triglyceride; $\mathrm{SBP}=$ systolic blood pressure; DBP = diastolic blood pressure.

previously for healthier HD patients carrying fewer comorbidities $[12,13]$.

The main barrier to introducing exercise in most dialyzed patients is lack of perseverance, which stems from insufficient knowledge about the advantages of physical activity. Such a patient attitude was the main reason for dropout during the study.

Twenty-one patients out of 29 (72.4\%) initially recruited completed the full 3 months of the exercise program. The main cause of dropping out in the group of 6 patients was lack of willingness to exercise. These patients did not differ in any feature from study subjects. They did not see the advantages of the program and finished it shortly after beginning.
In our study, exercise during dialysis lasting $50 \pm 19$ min did not cause serious complications. We observed transient mild incidents of hypo- and hypertension and no acute electrolyte disturbances (especially hyperpotassemia).

The implemented program of exercise has features of strength and also endurance training because of the substantial diversity of physical fitness in the study group, as mentioned above. As alluded in the methodology description, patients exhibited at the study onset significant variation in the muscle strength used during cycling. For that reason two different tests (6MWT and assessment of peak torque in the knee joint) were applied to estimate the influence of exercise on endurance and strength of dialysis patients. 
Taking Bohannon's norms for the general population, walking speed of our patients was much slower than agematched healthy people ( 6.66 vs. $3.56 \mathrm{~km} / \mathrm{h}, 52 \%$ of age norms) [14]. The ESRD population is as physically limited as patients with other chronic diseases. For example, speed gain by patients with heart failure NYHA III/IV waiting for heart transplantation in the study of Karapolat et al. [15] was $3.2 \mathrm{~km} / \mathrm{h}$. In another group of patients (patients with chronic obstructive lung disease), the indoor walking speed after converting the unit of measure was $3.94 \mathrm{~km} / \mathrm{h}$ [16].

The magnitude of improvement in 6MWT after the exercise program was not large (4\%), but it should be stressed that even a small improvement by old patients (average age 64 years) with a significant number of comorbidities could significantly improve self-care and functional independence, as was underlined by Cowen et al. [17].

Improvement of muscle function was also achieved in objective tests - assessment of peak torque in the knee joint. Statistically significant improvement was reached at an angular velocity of $60 \%$, and the maximal extension peak torque in the knee joint rose by $7 \%(69.77 \mathrm{Nm}$ before and $75.43 \mathrm{Nm}$ after cycle training, $\mathrm{p}=0.01$ ) and at an angular velocity of $300 \%$ by $4 \%(42.50 \mathrm{Nm}$ before and 44.11 $\mathrm{Nm}$ after cycle training, $\mathrm{p}=0.04)$. These pre- and postrehabilitation values are also worse compared to an agedmatched normal population from Bohannon norms and additionally confirm impairment of muscle function in ESRD patients. Likewise, in the aforementioned 6MWT the improvement was also not large, but indicates positive trends which probably would be better in the case of more intensive and longer training, as in the study by Headley et al. [12]. They reported $12.7 \%$ increase in maximal peak torque in the knee joint at an angular velocity of $90 \%$ (at baseline 123-139 Nm) after 12-week resistance training performed by HD patients. These data indicate how important the role played by rehabilitation is to maintain good physical condition.

There are a few studies showing additional beneficial effects of exercise on typical complications appearing in dialysis patients, namely better blood pressure control [18], malnutrition diminishment [19] and chronic inflammation amelioration [20]. Such positive effects did not occur in our study group, probably due to old age and a higher burden of comorbidities requiring application of a less intensive exercise program than was used in the reports concerning younger HD patients.

We are aware that this is a pilot study with a limited number of included patients and lacking a comparative control group, but at the same time we believe that the results support wider implementation of physical exercise in HD programs, especially in the elderly, with the aim of decreasing the very excessive rate of disabled patients with ESRD on HD [7].

\section{Conclusions}

Cycling during HD is a safe method to increase the strength of lower extremities and walking ability of older ESRD patients with many comorbidities. It could be easily applied in HD centers.

\section{Acknowledgments}

We would like to thank Dr. Waclaw Kopec for help with the biochemical analysis. This research was supported in part by an educational grant of the Medical University of Wroclaw.

\section{References}

1 Fitts SS, Guthrie MR, Blagg CR: Exercise coaching and rehabilitation counseling improve quality of life for predialysis and dialysis patients. Nephron 1999;82:115-121.

${ }_{2}$ Kettner-Melsheimer A, Weiss M, Huber W: Physical work capacity in chronic renal disease. Int J Artif Organs 1987;10:23-30.

3 Kutner NG, Zhang R, McClellan WM: Patient-reported quality of life early in dialysis treatment: effects associated with usual exercise activity. Nephrol Nurs J 2000;27:357367.
4 Beusterien KM, Nissenson AR, Port FK, Kelly M, Steinwald B, Ware JE Jr: The effects of recombinant human erythropoietin on functional health and well-being in chronic dialysis patients. J Am Soc Nephrol 1996;7: 763-773.

5 Moore GE, Parsons DB, Stray-Gundersen J, Painter PL, Brinker KR, Mitchell JH: Uremic myopathy limits aerobic capacity in hemodialysis patients. Am J Kidney Dis 1993;22: 277-287.
6 O’Hare AM, Tawney K, Bacchetti P, Johansen KL: Decreased survival among sedentary patients undergoing dialysis: results from the dialysis morbidity and mortality study wave 2. Am J Kidney Dis 2003;41:447-454.

-7 Altintepe L, Levendoglu F, Okudan N, Guney I, Savas Cilli A, Ugurlu H, Tonbul Z, Gokbel H, Turk S: Physical disability, psychological status, and health-related quality of life in older hemodialysis patients and agematched controls. Hemodial Int 2006;10: 260-266. 
8 Johansen KL, Sakkas GK, Doyle J, Shubert T, Dudley RA: Exercise counseling practices among nephrologists caring for patients on dialysis. Am J Kidney Dis 2003;41:171-178.

-9 Demers C, McKelvie RS, Negassa A, Yusuf S, RESOLVD Pilot Study Investigators: Reliability, validity, and responsiveness of the six-minute walk test in patients with heart failure. Am Heart J 2001;142:698-703.

10 Redelmeier DA, Bayoumi AM, Goldstein RS, Guyatt GH: Interpreting small differences in functional status: the Six Minute Walk test in chronic lung disease patients. Am J Respir Crit Care Med 1997;155:1278-1282.

11 Fitts SS, Guthrie MR: Six-minute walk by people with chronic renal failure. Assessment of effort by perceived exertion. Am J Phys Med Rehabil 1995;74:54-58.

12 Headley S, Germain M, Mailloux P, Mulhern J, Ashworth B, Burris J, Brewer B, Nindl BC, Coughlin M, Welles R, Jones M: Resistance training improves strength and functional measures in patients with end-stage renal disease. Am J Kidney Dis 2002;40:355-364.
3 Painter P, Carlson L, Carey S, Paul SM, Myll $\mathrm{J}$ : Physical functioning and health-related quality-of-life changes with exercise training in hemodialysis patients. Am J Kidney Dis 2000;35:482-492.

14 Bohannon RW: Comfortable and maximum walking speed of adults aged 20-79 years: reference values and determinants. Age Ageing 1997;26:15-19.

15 Karapolat H, Eyigor S, Zoghi M, Nalbantgil S, Yagdi T, Durmaz B, Ozbaran M: Health related quality of life in patients awaiting heart transplantation. Tohoku J Exp Med 2008;214:17-25.

16 Brooks D, Solway S, Weinacht K, Wang D, Thomas S: Comparison between an indoor and an outdoor 6-minute walk test among individuals with chronic obstructive pulmonary disease. Arch Phys Med Rehabil 2003. 84:873-876.
Cowen TD, Huang CT, Lebow J, DeVivo MJ, Hawkins LN: Functional outcomes after inpatient rehabilitation of patients with endstage renal disease. Arch Phys Med Rehabil 1995;76:355-359.

18 Miller BW, Cress CL, Johnson ME, Nichols $\mathrm{DH}$, Schnitzler MA: Exercise during hemodialysis decreases the use of antihypertensive medications. Am J Kidney Dis 2002;39: 828-833.

19 Załuska A, Załuska WT, Bednarek-Skublewska A, Ksiazek A: Nutrition and hydration status improve with exercise training using stationary cycling during hemodialysis (HD) in patients with end-stage renal disease (ESRD). Ann Univ Mariae Curie Sklodowska Med 2002;57:342-346.

20 Castaneda C, Gordon PL, Parker RC, Uhlin KL, Roubenoff R, Levey AS: Resistance training to reduce the malnutrition-inflammation complex syndrome of chronic kidney disease. Am J Kidney Dis 2004;43:607-616. 